\title{
A NOTE ON ELLIPSOIDAL SHAPE AND GRAVITATIONAL POTENTIAL FIRST ORDER RELATIONSHIPS IN PLANETARY GEODESY
}

\author{
G. Balmino \\ Groupe de Recherches de Geodesie Spatiale, \\ O.M.P. \\ 14, Avenue Edouard Belin, 31400 Toulouse, France \\ Tel.: +33 (0)561332889 \\ E-mail: balmino@dtp.obs-mip.fr
}

\begin{abstract}
We generalize the basic first order Clairaut formula to the case of a triaxial ellipsoid subject to rotational and tidal deformations from another body. We also prove and put together some relationships involving the gravitational static harmonics and the secular Love number of degree two, which are of wide use in planetary geodesy though difficult to find in textbooks.
\end{abstract}

Keywords : Clairaut theory. Ellipsoidal shape. Gravity field harmonics. Love numbers.

\section{INTRODUCTION}

The shape of large bodies in the solar system can be well approximated by ellipsoids of revolution (case of the planets) or by triaxial ellipsoids (case of many natural satellites). Such shapes result from the rotational deformation of the body on the one hand, and from the tidal distortion due to the gravitational field of a parent body (in most cases the mother planet sometimes the Sun) on the other hand. It is therefore necessary to extend the classical Clairaut theory which applies to an ellipsoid (E) of revolution (with equatorial and polar radii $a, c$ respectively) and relates its geometrical flattening $(\alpha)$ and second degree gravitational harmonic $\left(C_{20}\right)$ to the body mean angular rotation velocity $(\omega)$, size (via $a$ ) and mass $(M)$, assuming that the boundary surface of $(\mathrm{E})$ is an equipotential of $W$, the sum of the gravitational potential $U$ and centrifugal potential $C$, that is:

$$
\begin{gathered}
W=U+C \\
U=\frac{G M}{r}+\frac{G M}{a}\left(\frac{a}{r}\right)^{3} C_{20} P_{2}(\sin \varphi) \\
C=\frac{1}{2} \omega^{2} r^{2} \cos ^{2} \varphi \\
=\frac{1}{3} \omega^{2} r^{2}\left[1-P_{2}(\sin \varphi)\right]
\end{gathered}
$$

$W$ is called gravity potential (gravity being the sum of the gravitational and centrifugal effects - the acceleration which is indeed felt at the surface of the body or at any point bound to the body). $G$ is the Newtonian gravitational constant, $r$ and $\varphi$ are the radius vector and planetocentric latitude respectively; the third spherical coordinate (the longitude $\lambda$ ) is not 
involved in this simple problem. $C_{20}$ is often replaced by $-J_{2}$, and $P_{2}(\sin \varphi)$ is the usual Legendre polynomial of degree two, equal to $3 / 2 \sin ^{2} \varphi-1 / 2$. The coordinate system rotates with (E), its origin is the geometrical center of the ellipsoid and coincides with its center of mass, implying that there are no terms of degree one in the above.

$\alpha$ is the usual polar flattening, equal to $(a-c) / a$ and is assumed to be a small quantity as well as $C_{20}$ and $m=\omega^{2} a^{3} / G M$. On (E) the radius vector is: $r=a\left(1-\alpha \sin ^{2} \varphi\right)$, which is often used to define the so-called Clairaut spheroid.

To first order in all small parameters Clairaut formula is:

$$
\alpha=\frac{m}{2}-\frac{3}{2} C_{20}
$$

This is independent of the density distribution inside (E), which is not the case e.g. of Mac Laurin ellipsoids which are homogeneous. This is one result of the solution of the fundamental problem of finding figures of equilibrium and related questions in which Poincaré (1902) made pioneering contributions. Very detailed developments, with higher order formulas, can be found more recently in Chandrasekhar (1969), Melchior (1971) and Moritz (1990), to quote a few. Hubbard (1984) treated specific applications in planetology.

It is of interest for the next sections of this paper to give the value $W_{0}$ of the constant total potential on (E). Using (2), (3) and (4) one finds :

$$
W_{0}=\frac{G M}{a}\left(1+\alpha+C_{20}\right)
$$

A more frequently used (first order) formula is obtained by introducing the sphere of radius $R$ which has the same volume as the ellipsoid, since one usually determines $R$ from telescopic observations or from navigation data and pictures taken from a spacecraft orbiting or flying by the studied body. Therefore $R^{3}=\mathrm{a}^{2} c=a^{3}(1-\alpha), 1 / a \approx 1 / R(1-\alpha / 3)$ and, by taking account of (4) and approximating $m$ by $\omega^{2} R^{3} / G M$, we get:

$$
W_{0}=\frac{G M}{R}(1+m / 3)
$$

The use of $R$ instead of $a$ also implies that on (E) we have:

$$
r=R\left[1-\frac{2}{3} \alpha P_{2}(\sin \varphi)\right]=R\left[1-\left(\frac{m}{3}-C_{20}\right) P_{2}(\sin \varphi)\right]
$$

In the following we will revisit the theory in two cases:

- the body, still rotating but isolated in space, is approximated by a triaxial ellipsoid, a shape which may be inherited from a past situation (for instance in the presence of a disturbing body, ignored at this stage); one assumes that it is still a level surface,

- the body is an equilibrium triaxial ellipsoid, a shape due to distortion produced by rotation and tidal forces exerted by a parent planet, or the Sun, resulting in a spin-orbit coupling state the most common case being the body in synchronous rotation with respect to its primary.

\section{ROTATING TRIAXIAL ELLIPSOID ISOLATED IN SPACE}

Let $a, b, c$ be the three axes of the ellipsoid (E), with $a \geq b, a \geq c$ (but $b \geq c$ or $b<c$ ), and $\omega$ its angular velocity as above. The reference system origin is again the geometrical center of $(\mathrm{E})-$ 
also its center of mass, the third axis coincides with the (mean) rotation axis (of length $2 c$ ), the first axis (origin of longitudes $\lambda$ ) is along the longest axis of $(\mathrm{E})$.

The gravity potential is $W$, expressed in spherical coordinates as :

$$
W=\frac{G M}{r}+\frac{G M}{a}\left(\frac{a}{r}\right)^{3}\left[C_{20} P_{20}(\sin \varphi)+C_{22} P_{22}(\sin \varphi) \cos 2 \lambda\right]+\frac{1}{2} \omega^{2} r^{2} \cos ^{2} \varphi
$$

where $P_{22}(\sin \varphi)=3 \cos ^{2} \varphi$, and $C_{22}$ is the gravitational harmonic coefficient of degree and order 2 (the associated $S_{22}$ harmonic is zero in our reference system). All other quantities are identical to those previously defined.

At the surface of $(\mathrm{E})$, we have (from its cartesian equation):

$$
\frac{r^{2}}{a^{2}}\left(1+\frac{2 \alpha-\alpha^{2}}{(1-\alpha)^{2}} \sin ^{2} \varphi+\frac{2 \varepsilon-\varepsilon^{2}}{(1-\varepsilon)^{2}} \cos ^{2} \varphi \sin ^{2} \lambda\right)=1
$$

where $\alpha$ is the polar flattening (with respect to the largest [equatorial] axis), and $\varepsilon$ is the equatorial flattening, that is:

$$
\alpha=\frac{a-c}{a} \quad \text { and } \quad \varepsilon=\frac{a-b}{a}
$$

From our hypothesis, both flattenings are positive. To first order in these quantities, we write:

$$
r=a\left(1-\alpha \sin ^{2} \varphi-\varepsilon \cos ^{2} \varphi \sin ^{2} \lambda\right)
$$

which may be considered defining a triaxial spheroid.

We assume that $\alpha$ and $\varepsilon$ are small enough so that equations (8) and (9) are sufficient to represent the gravity field and the radius vector of the ellipsoid; otherwise more accurate (and complex) formulas could be used (Balmino, 1994).

Introducing $\widetilde{W}=W /(G M / a)$ and again $m=\omega^{2} a^{3} / G M$ we then find:

$$
\widetilde{W}=1+\alpha+C_{20}+\cos ^{2} \varphi\left(\frac{\varepsilon}{2}-\alpha-\frac{3}{2} C_{20}+\frac{m}{2}\right)+\cos ^{2} \varphi \cos 2 \lambda\left(3 C_{22}-\frac{\varepsilon}{2}\right)
$$

Sufficient conditions for this function to be independent of $\varphi$ and $\lambda$ are:

$$
\begin{gathered}
\frac{\varepsilon}{2}-\alpha-\frac{3}{2} C_{20}+\frac{m}{2}=0 \\
\frac{\varepsilon}{2}=3 C_{22}
\end{gathered}
$$

This implies another (not independent) relation not involving $\varepsilon$ :

$$
\alpha-\frac{m}{2}=-\frac{3}{2} C_{20}+3 C_{22}
$$

This generalizes Clairaut's equation (4). The potential on (E) has the constant value: $W_{0}=(G M / a)\left(1+\alpha+C_{20}\right)$, which is identical to (5) and which can be transformed by introducing the sphere of radius $R=(a b c)^{1 / 3} \cong a(1-\alpha / 3-\varepsilon / 3)$ and by again approximating $m$ by $\omega^{2} R^{3} / G M$; this results in: 


$$
W_{0}=\frac{G M}{R}\left(1+2 \alpha / 3+C_{20}-\varepsilon / 3\right)
$$

Taking (14) into account this reduces to:

$$
W_{0}=\frac{G M}{R}(1+m / 3)
$$

that is exactly equation (6).

\section{TRIAXIAL ELLIPSOID RESULTING FROM SPIN AND TIDAL DISTORTION}

We take the same ellipsoid as in the previous section but we here assume that it is in orbit around a parent body $\left(\mathrm{P}^{*}\right)$, approximated by a point mass $P^{*}$ of mass $M^{*}$. Then any point $P$ on (E) is (also) subject to the disturbing (tidal) potential $U^{*}$ :

$$
U^{*}=\frac{G M^{*}}{r^{*}} \sum_{\ell=2}^{\infty}\left(\frac{r}{r^{*}}\right)^{\ell} P_{\ell}(\cos \psi)
$$

where $P_{\ell}$ is the Legendre polynomial of degree $\ell, r^{*}$ is the distance of $P^{*}$ from the origin and $\psi$ is the angular distance between $P$ and $P^{*}$.

Now we assume that the orbit of $(\mathrm{E})$ around $\left(\mathrm{P}^{*}\right)$ is almost equatorial and circular - that is its eccentricity and all acting perturbations are rather small, implying that $r^{*}$ is close to a constant $A^{*}$ (the semi-major axis of the orbit), and that (E) is in a spin-orbit resonant state with respect to $\left(\mathrm{P}^{*}\right)$. Consequently the latitude $\varphi^{*}$ of $P^{*}$ is zero and its longitude $\lambda^{*}$ remains linear in the reference frame rotating with (E). If $n^{*}$ is the orbital mean motion we have $\lambda^{*}=-\left(\omega-n^{*}\right) t$ and $\omega / n^{*}=h / p \geq 1$ ( $t$ is the time; $h, p$ are integers) due to the tidal (deceleration) process for instance this ratio is $3 / 2$ for Mercury. In the case of synchronous rotation we have $\lambda^{*}=0$ i.e. $P^{*}$ is located on the $x$-axis, the origin of longitudes on $(\mathrm{E})$. Using third Kepler's law we have:

$$
\omega^{2}=(h / p)^{2} G\left(M^{*}+M\right) / A^{* 3}
$$

which simplifies into

$$
\omega^{2}=(h / p)^{2} G M^{*} / A^{* 3}
$$

if $M<<M^{*}$, an assumption verified for all planet-satellite or sun-planet association in the solar system.

Usually $r^{*}$ is so much larger than $r$ that the first term (of degree two) in equation (16) is sufficient. Besides, since the orbital motion is close to circular, we take the average of $1 / r^{* 3}$ equal to $1 / A^{* 3}$; and since $\cos \psi=\cos \varphi \cos \left(\lambda-\lambda^{*}\right)$ we have:

$$
U^{*}=\frac{G M^{*}}{r^{* 3}} r^{2}\left[\frac{3}{2} \cos ^{2} \varphi \cos ^{2}\left(\lambda-\lambda^{*}\right)-\frac{1}{2}\right]
$$

Finally the total gravity potential, $W=U+C+U^{*}$ is equal to:

$$
\begin{aligned}
W=\frac{G M}{r}+\frac{G M}{a}\left(\frac{a}{r}\right)^{3}[ & \left.C_{20}\left(1-\frac{3}{2} \cos ^{2} \varphi\right)+3 C_{22} \cos ^{2} \varphi \cos 2 \lambda\right]+\frac{1}{2} \omega^{2} r^{2} \cos ^{2} \varphi \\
+ & \frac{G M^{*}}{A^{* 3}} r^{2}\left(\frac{3}{2} \cos ^{2} \varphi \frac{1+\cos 2\left(\lambda-\lambda^{*}\right)}{2}-\frac{1}{2}\right)
\end{aligned}
$$


As before we consider $\widetilde{W}=W /(G M / a)$ which, by means of (10), is easily put in a form analogous to (11):

$$
\begin{aligned}
\widetilde{W}=1+\alpha+C_{20}-\frac{q^{*}}{2}+\cos ^{2} \varphi( & \left.\frac{\varepsilon}{2}-\alpha-\frac{3}{2} C_{20}+\frac{m}{2}+\frac{3}{4} q^{*}\right) \\
& +\cos ^{2} \varphi \cos 2 \lambda\left(3 C_{22}-\frac{\varepsilon}{2}+\frac{3}{4} q^{*} \cos 2 \lambda^{*}\right) \\
& +\frac{3}{4} q^{*} \cos ^{2} \varphi \sin 2 \lambda \sin 2 \lambda^{*}
\end{aligned}
$$

where we have introduced the tidal parameter $q^{*}=\left(M^{*} / M\right)\left(a / A^{*}\right)^{3}$. It is obvious that the surface of (E) can be an equipotential only if $\lambda^{*}=0$ that is in the synchronous rotation case: $h / p=1$, which will be assumed in the remaining of this section. From the definition of $m$ and from (17) we then obtain the important relation:

$$
q^{*}=m
$$

The equipotential condition yields:

$$
\begin{gathered}
W_{0}=\frac{G M}{a}\left(1+\alpha+C_{20}-q^{*} / 2\right) \\
\frac{\varepsilon}{2}-\alpha-\frac{3}{2} C_{20}+\frac{m}{2}+\frac{3}{4} q^{*}=0 \\
\frac{\varepsilon}{2}=3 C_{22}+\frac{3}{4} q^{*}
\end{gathered}
$$

As we did it in section 2, using $R=a(1-\alpha / 3-\varepsilon / 3)$ we can easily transform (22) in:

$$
W_{0}=\frac{G M}{R}\left(1+2 \alpha / 3+C_{20}-\varepsilon / 3-q^{*} / 2\right)
$$

According to (23) this reduces to:

$$
W_{0}=\frac{G M}{R}(1+m / 3)
$$

which is again equation (6)!

From (23) and (24) we derive another (generalized) Clairaut-type equation:

$$
\alpha-\frac{m}{2}=-\frac{3}{2} C_{20}+3 C_{22}+\frac{3}{2} q^{*}
$$

Using (21) we obtain the simpler formula:

$$
\alpha=-\frac{3}{2} C_{20}+3 C_{22}+2 m
$$

We can go a step further and find another relationship by interpreting the gravitational potential change resulting from the deformation of the sphere (of radius $R$ ) into this triaxial ellipsoid, in the framework of Love theory (Melchior, ibid.).

Assuming the deformation is solely due to the rotation of $(\mathrm{E})$ and the tidal perturbation of $\left(\mathrm{P}^{*}\right)$ we start from the disturbing potential: 


$$
\begin{aligned}
\Delta W & =C+U^{*} \\
& =\frac{1}{2} \omega^{2} r^{2} \cos ^{2} \varphi+\frac{G M^{*}}{A^{* 3}} r^{2}\left(\frac{3}{2} \cos ^{2} \varphi \frac{1+\cos 2 \lambda}{2}-\frac{1}{2}\right)
\end{aligned}
$$

To apply Love elasticity theory we need to write it in terms of spherical harmonics only; using $P_{2}(\sin \varphi)$ as in (2) and (3) and $P_{22}(\sin \varphi)$ as in (8) we find:

$$
\Delta W=a_{00}(r)+a_{20}(r) P_{2}(\sin \varphi)+a_{22}(r) P_{22}(\sin \varphi) \cos 2 \lambda
$$

with

$$
\begin{gathered}
a_{00}(r)=r^{2} \omega^{2} / 3 \\
a_{20}(r)=-\frac{r^{2}}{3}\left(\omega^{2}+\frac{3}{2} \frac{G M^{*}}{A^{* 3}}\right) \\
a_{22}(r)=\frac{r^{2}}{4} \frac{G M^{*}}{A^{* 3}}
\end{gathered}
$$

At the surface of the sphere we put $r=R$. The term $a_{00}(R)$ gives rise to a purely radial deformation which is ignored in our problem (it slightly changes the mean radius $R$ ). The remaining second degree terms, $\Delta W_{2}$, deform the sphere and change its gravitational potential on its surface by the amount:

$$
\delta W_{2}(R, \varphi, \lambda)=k_{2 S} \Delta W_{2}(R, \varphi, \lambda)
$$

where $k_{2 S}$ is the secular Love number of degree two since we are here dealing with the tidal distortion acquired (and maintained) over a long time ( $k_{2 S}=k_{f}=1.5$ for a homogeneous fluid).

The external $(r>R)$ gravitational potential change is therefore (according to a classical Dirichlet theorem):

$$
\delta W_{2}(r, \varphi, \lambda)=\left(\frac{R}{r}\right)^{3} k_{2 S}\left[a_{20}(R) P_{2}(\sin \varphi)+a_{22}(R) P_{22}(\sin \varphi) \cos 2 \lambda\right]
$$

It is then compared to the non central part of $U$, that is (with $a \approx R$ ):

$$
\delta U=\frac{G M}{R}\left(\frac{R}{r}\right)^{3}\left[C_{20} P_{20}(\sin \varphi)+C_{22} P_{22}(\sin \varphi) \cos 2 \lambda\right]
$$

from which we readily find:

$$
\begin{aligned}
& C_{20}=k_{2 S} \frac{R}{G M} a_{20}(R) \\
& C_{22}=k_{2 S} \frac{R}{G M} a_{22}(R)
\end{aligned}
$$

Introducing $m$ and $q^{*}$ (again with $a \approx R$ ) we obtain:

$$
\begin{aligned}
& C_{20}=-k_{2 S}\left(\frac{1}{3} m+\frac{1}{2} q^{*}\right) \\
& C_{22}=\frac{1}{4} k_{2 S} q^{*}
\end{aligned}
$$


These equations are valid if one assumes that the gravitational potential of $(E)$ is solely due to the spin-tidal distorsion. We also note that the first one generalizes the well-known relationship $k_{2 S}=-3 C_{20} / m$ which applies to the giant planets.

Finally if we take into account (21) the above equations can be rewritten:

$$
\begin{aligned}
& C_{20}=-\frac{5}{6} k_{2 S} m \\
& C_{22}=\frac{1}{4} k_{2 S} m
\end{aligned}
$$

from which we derive another known relationship between $C_{20}$ and $C_{22}$ :

$$
C_{20}=-\frac{10}{3} C_{22}
$$

This may further be used to transform equation (27) into:

$$
\alpha-2 m=-\frac{12}{5} C_{20}=8 C_{22}
$$

\section{CONCLUSION}

We have found some new relations between the shape parameters of a triaxial ellipsoid and its second degree gravitational harmonics, which are a kind of generalisation of Clairaut fundamental equation. These equations are all good to first order in all involved small parameters but should suffice for many investigations. Depending on the "best" observed quantities, other parameters can be computed from these equations; for instance, one may determine $a, b, c$ from observed (or theoretical) values of $G M, R, \omega, C_{20}, C_{22}$, or estimate the secular Love number of degree two which is of great importance for models of the interior.

\section{REFERENCES}

Balmino, G. (1994), Gravitational potential harmonics from the shape of an homogeneous body, Celestial Mechanics \& Dynamical Astronomy, Vol. 60, N. 3, pp. 331-364.

Chandrasekhar, S. (1969), Ellipsoidal Figures of Equilibrium, Yale Univ. Press, New Haven.

Hubbard, W. B. (1984), Planetary Interiors, Van Nostrand-Reinhold Ed. , New-York.

Melchior, P. (1971), Physique et Dynamique Planétaire, Vol. 2 \& 3, Vander Ed. , Louvain and Brussels.

Moritz, H. (1990), The Figure of the Earth: Theoretical Geodesy and the Earth's Interior, Wichmann Ed. , Karlsruhe.

Poincaré, H. (1902), Figures d'équilibre d'une masse fluide, Gauthiers-Villars Ed., Paris.

Received : 2008-01-28,

Reviewed : 2008-02-29,

Accepted : 2008-06-04. 